\title{
Changes in the gray and white matter of patients with ischemic-edematous insults after traumatic brain injury
}

\author{
Hakseung Kim, PhD, ${ }^{1}$ Young-Tak Kim, MEng,, Eun-Suk Song, MEng, ${ }^{1}$ Byung C. Yoon, MD, PhD, ${ }^{2}$ \\ Young Hun Choi, MD, ${ }^{3}$ Keewon Kim, MD, PhD, ${ }^{4}$ and Dong-Joo Kim, PhD ${ }^{1}$
}

${ }^{1}$ Department of Brain and Cognitive Engineering, Korea University, Seongbuk-gu, Seoul, South Korea; ${ }^{2}$ Department of Radiology, Stanford University School of Medicine, Stanford, California; and Departments of ${ }^{3}$ Radiology and ${ }^{4}$ Rehabilitation, Seoul National University Hospital, College of Medicine, Jongno-gu, Seoul, South Korea

\begin{abstract}
OBJECTIVE Gray matter (GM) and white matter (WM) are vulnerable to ischemic-edematous insults after traumatic brain injury (TBI). The extent of secondary insult after brain injury is quantifiable using quantitative CT analysis. One conventional quantitative CT measure, the gray-white matter ratio (GWR), and a more recently proposed densitometric analysis are used to assess the extent of these insults. However, the prognostic capacity of the GWR in patients with TBI has not yet been validated. This study aims to test the prognostic value of the GWR and evaluate the alternative parameters derived from the densitometric analysis acquired during the acute phase of TBI. In addition, the prognostic ability of the conventional TBI prognostic models (i.e., IMPACT [International Mission for Prognosis and Analysis of Clinical Trials in TBI] and CRASH [Corticosteroid Randomisation After Significant Head Injury] models) were compared to that of the quantitative CT measures.
\end{abstract}

METHODS Three hundred patients with TBI of varying ages (92 pediatric, 94 adult, and 114 geriatric patients) and admitted between 2008 and 2013 were included in this retrospective cohort study. The normality of the density of the deep GM and whole WM was evaluated as the proportion of CT pixels with Hounsfield unit values of 31-35 for GM and 26-30 for WM on CT images of the entire supratentorial brain. The outcome was evaluated using the Glasgow Outcome Scale (GOS) at discharge (GOS score $\leq 3, \mathrm{n}=100$ ).

RESULTS Lower proportions of normal densities in the deep GM and whole WM indicated worse outcomes. The proportion of normal WM exhibited a significant prognostic capacity (area under the curve $[A U C]=0.844$ ). The association between the outcome and the normality of the WM density was significant in adult (AUC $=0.792$ ), pediatric (AUC = 0.814 ), and geriatric (AUC $=0.885$ ) patients. In pediatric patients, the normality of the overall density and the density of the GM were indicative of the outcome (AUC = 0.751). The average GWR was not associated with the outcome (AUC $=0.511$ ). IMPACT and CRASH models showed adequate and reliable performance in the pediatric and geriatric groups but not in the adult group. The highest overall predictive performance was achieved by the densitometry-augmented IMPACT model (AUC = 0.881).

CONCLUSIONS Both deep GM and WM are susceptible to ischemic-edematous insults during the early phase of TBI. The extent of the secondary injury was better evaluated by analyzing the normality of the deep GM and WM rather than by calculating the GWR.

https://thejns.org/doi/abs/10.3171/2018.5.JNS172711

KEYWORDS traumatic brain injury; computed tomography; densitometry; gray matter; white matter

$\mathrm{T}$ Raumatic brain injury (TBI) is widely recognized as one of the most significant causes of mortality and morbidity, and due to its high incidence, TBI is often referred to as a "silent epidemic."10,26 Timely interventions for secondary injuries are extremely important in patients with head injuries and require an early assessment of the injury severity. Injury severity is commonly evaluated using different scales and parameters, such as the Glasgow Coma Scale (GCS), ${ }^{29}$ among others. ${ }^{1}$ However, these measures are not always feasible to perform,

ABBREVIATIONS AUC = area under the curve; $C C=$ corpus callosum; $C N=$ caudate nucleus; $C R A S H=$ Corticosteroid Randomisation After Significant Head Injury; dGx = density normality of deep GM; dWx = density normality of deep WM; GCS = Glasgow Coma Scale; GM = gray matter; GOS = Glasgow Outcome Scale; GWR = graywhite matter ratio; HU = Hounsfield unit; IMPACT = International Mission for Prognosis and Analysis of Clinical Trials in TBI; IQR = interquartile range; PLIC = posterior limb of the internal capsule; $\mathrm{PU}=$ putamen; $\mathrm{ROC}=$ receiver operating characteristic; $\mathrm{ROI}=$ region of interest; $\mathrm{TBI}=$ traumatic brain injury; WM = white matter.

SUBMITTED October 26, 2017. ACCEPTED May 10, 2018.

INCLUDE WHEN CITING Published online November 2, 2018; DOI: 10.3171/2018.5.JNS172711. 
particularly when the patient is intubated. The application of neuroimaging techniques, particularly $\mathrm{CT}$, during the acute phase of an injury can compensate for this limitation, and these techniques are widely used to define the cause and extent of neurological injury. ${ }^{38}$

Nearly all complications after TBI provoke secondary ischemia, ${ }^{16}$ and the extent of the secondary ischemicedematous insult is often assessed via CT during the acute phase of TBI. The extent of cerebral ischemia often manifests as the loss of gray matter (GM) and white matter (WM) differentiation on brain CT.9 This pathoanatomical feature effectively predicts poor outcomes after hypoxicischemic injury, ${ }^{8,9}$ although non-negligible inter- and intraobserver variability exists because the observed decreases in the density of the GM and/or WM on CT are often very subtle. ${ }^{3}$ Minimization of inter- and intraobserver variability in the interpretation of CT images remains an important issue, which might be addressed by a quantitative CT analysis.

The gray-white matter ratio (GWR) is used to quantitatively measure the degree of the loss of GM-WM differentiation, ${ }^{37}$ which reduces the inter- and intraobserver variability. Lower GWRs are associated with worse outcomes following cardiac arrest in adult and pediatric patients; $5,21,27,33$ however, the prognostic capacity of the GWR in TBI has not been reported to date. Furthermore, according to previous studies, $3,21,37$ the significant decrease in GM density mainly contributes to the high predictive capacity of the GWR, whereas the density of the WM remains relatively stable. However, a decrease in the WM density has been reported in patients with $\mathrm{TBI},{ }^{42}$ which may lower the efficacy of the GWR in determining the prognosis of patients with TBI. This limitation of the GWR might be compounded by the following observations: 1 ) the derivation of GWR requires the manual designation of a small region of interest (ROI), and 2) a consensus on measuring GWR is not yet available. ${ }^{18}$

A more recent approach for a quantitative CT analysis of patients with an acquired brain injury is densitometric analysis. ${ }^{11,12}$ Instead of relying on specific, localized ROIs to investigate pathological changes in the density of brain tissues, this approach measures changes in the overall density of the entire brain in a completely automated manner. ${ }^{11,12}$ Of particular interest, the densitometric analysis enables a quantitative examination of the parenchymal density distribution, thereby enabling subsequent measurements of the proportions of GM and WM showing a normal density. ${ }^{11,35}$ If both the deep GM and WM densities decrease in response to ischemic-edematous insults after TBI, then the quantification of the material density of these structures could significantly enhance the prognostic capacity of noncontrast CT images in patients with acute TBI.

This study aims to determine: 1) whether the GWR is effective in patients with TBI; 2) whether the densitometric parameters representing changes in the proportions of deep GM and WM are useful predictors of the outcomes of patients with TBI; and, finally, 3) whether the densitometric parameters could be incorporated into existing TBI prognostication models, namely, IMPACT (International Mission for Prognosis and Analysis of Clinical Trials in
TBI) ${ }^{34}$ and CRASH (Corticosteroid Randomisation After Significant Head Injury), ${ }^{23}$ thereby contributing to obtaining a better prognosis after TBI.

\section{Methods \\ Study Design and Setting}

The main objective of this retrospective cohort study was to evaluate the prognostic capacities of changes in the densities of deep GM and WM after TBI. Therefore, clinical information and noncontrast $\mathrm{CT}$ images that were initially acquired from patients with TBI within 24 hours of the emergency room admission were analyzed. Anonymized data (CT images, radiology reports, patient records, age, sex, etc.) from patients with TBI who were admitted between 2008 and 2013 and underwent CT examinations using the same imaging modality (Brilliance 64, Philips) were initially collected. CT examinations were performed on patients who experienced motor vehicle accidents, falls, collisions, headaches, vomiting, nausea, dizziness, seizures, concussions, confusion, etc. The use of the study material was approved by the IRB at Seoul National University Hospital. The requirement to obtain informed consent from the patients for the use of the materials was waived based on the retrospective nature of the study under the approval of the IRB. Patients were excluded from the analyses if any of the clinical information and/or CT images was missing or the parameters could not be derived.

\section{Selection of Participants}

The tissue density on the CT images was measured in Hounsfield units (HUs); lower density tissue exhibits a lower HU value and vice versa. Differences in the CT examination parameters (e.g., tube voltage, tube current, and CT scanner types) are major factors that cause variability in HU values. Additionally, the brain density is significantly affected by the age of the patients; for example, myelination is incomplete in neonates/pediatric patients younger than 2 years. ${ }^{15}$ The following inclusion criteria were applied to minimize possible sources of HU variability: 1) the same CT scanner type (Brilliance 64, Philips) was used, 2) the CT images were acquired within 24 hours of injury, 3) the tube voltage was $120 \mathrm{kVp}$, and 4) the tube current was $>150 \mathrm{~mA}$. In addition, patients were further excluded if 1) the etiology was not strictly traumatic, 2) the age of the patient at admission was younger than 2 years, and 3) the image contained a significant artifact. All images were acquired in the axial mode (image matrix size $512 \times 512$ ). Patients younger than 18 years were classified as pediatric, patients older than 65 years were classified as geriatric, and other patients were classified as adults. A total of 300 patients met these inclusion/exclusion criteria, were deemed eligible for further analyses, and were ultimately included in the study (Fig. 1).

\section{Derivation of GWR}

The extent of the ischemic-edematous insult in the GM and WM was evaluated by measuring the GWR and performing a densitometric analysis. GWRs were obtained using the method proposed by Lee et al. ${ }^{18}$ which derives 


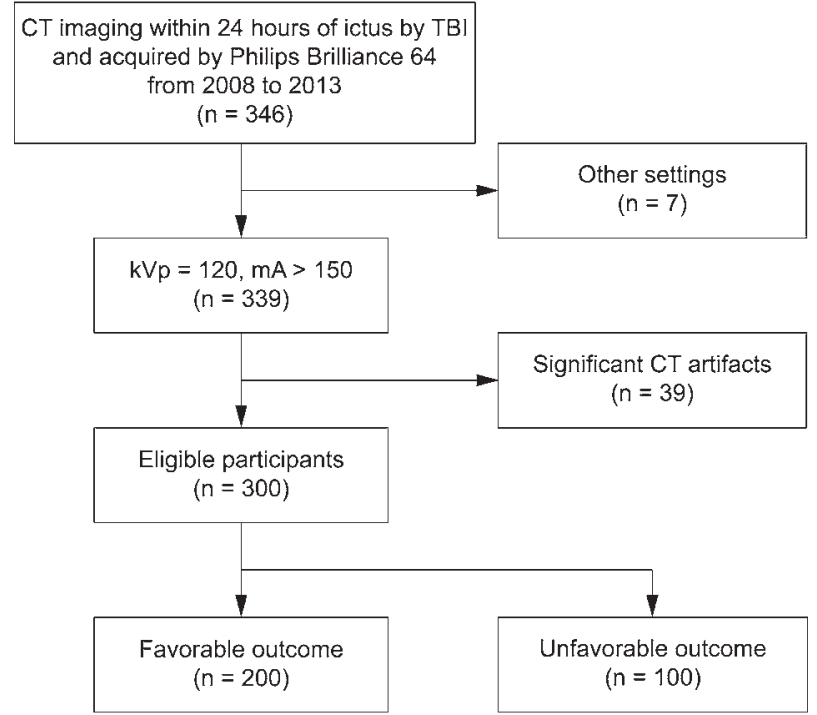

FIG. 1. Flowchart illustrating the patient selection process. In total, 300 patients with TBI were selected from an institutional database according to four inclusion criteria and three exclusion criteria.

"normalized" GWRs by averaging various GWR measures. ${ }^{3,14,17,21,37,40}$ A $10-\mathrm{mm}^{2}$ circular region of measurement was placed over the ROIs, and the HU values for the deep GM (caudate nucleus [CN] and putamen [PU]) and WM (posterior limb of the internal capsule [PLIC] and corpus callosum $[\mathrm{CC}]$ ), the medial cortex ( $\mathrm{MC} 1$ at the centrum semiovale and $\mathrm{MC} 2$ at high convexity levels) and the medial WM (MW1 at the centrum semiovale and MW2 at high convexity levels) were recorded. GWR parameters were calculated using the following equations: $\mathrm{CN} / \mathrm{PLIC}, \mathrm{PU} / \mathrm{PLIC}, \mathrm{GWR}_{\text {basal ganglia }}=(\mathrm{CN}+\mathrm{PU}) /(\mathrm{PLIC}$ $+\mathrm{CC}), \mathrm{GWR}_{\text {cerebrum }}=(\mathrm{MC1}+\mathrm{MC} 2) /(\mathrm{MW} 1+\mathrm{MW} 2)$, and average GWR $=\left(G_{\text {basal ganglia }}+\mathrm{GWR}_{\text {cerebrum }}\right) / 2$. The values for the GWR parameters were derived based on the consensus of two specialists (K.K. and D.J.K.) who were blinded to the outcome to minimize interrater variability. The derivation of the GWR was performed retrospectively and did not influence the management of the patients.

\section{Quantitative Densitometric Analysis}

A set of contiguous axial CT images (slice thickness 5 $\mathrm{mm}$ ) of the entire supratentorial region was designated the ROI for the densitometric analysis to achieve the objective of this study (Fig. 2). The analysis was conducted using an in-house software program implemented in JAVA (Oracle Inc.). ${ }^{11}$ The software allows the exclusion of minor intracranial entities (cranium, calcifications, etc.) from the analysis and accurately derives the density distribution of major intracranial entities (i.e., CSF, blood, and parenchyma), which have an HU range of 0-79.28

After calculating the density distribution, the density normality of GM and WM was derived. The GM typically exhibits HU values ranging from 30 to 40 , and the deep $\mathrm{GM}$ has a more narrow range of $\mathrm{HU}$ values $(31-35){ }^{2} \mathrm{Ad}-$ ditionally, the WM density ranges from 20 to $30,{ }^{39}$ which overlaps with the HU value of the CSF $(15-25 \mathrm{HUs}){ }^{43}$
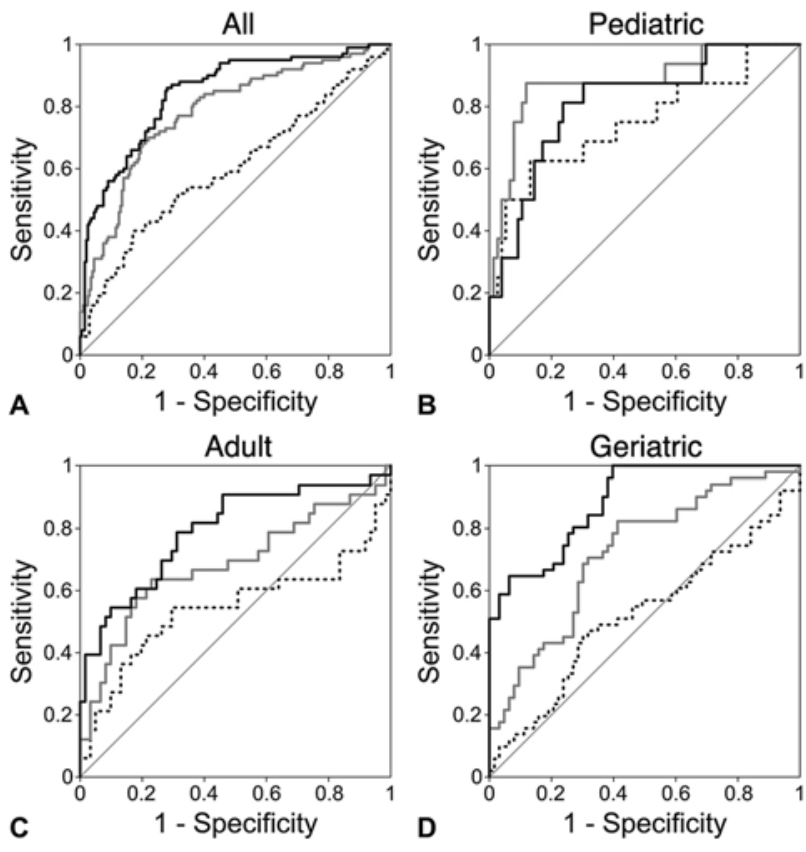

C

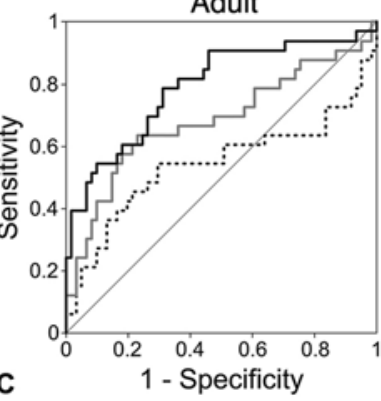

$-\mathrm{dWx} \cdots \mathrm{dGx}-\mathrm{dWx}+\mathrm{dGx}$

FIG. 2. Predictive power of the densitometric parameters in assessing the prognostic capacity of unfavorable outcomes. ROC curves show both the sensitivity and specificity of the densitometric parameters in assessing the prognosis of unfavorable outcomes in all (A), pediatric (B), adult (C), and geriatric (D) patients.

Therefore, in this study, the density normality of the deep GM and WM (designated "dGx" and "dWx," respectively) was defined as the proportion of pixels with $\mathrm{HU}$ values of 31-35 (dGx) and 26-30 (dWx) among all pixels of the supratentorial brain.

\section{Outcomes}

The outcome was evaluated using the Glasgow Outcome Scale (GOS) at hospital discharge, which was measured by the attending clinician. Good or moderate disability (GOS scores of 4 or 5) were deemed favorable, whereas severe disability, a persistent vegetative state, or death (GOS scores 1-3) were considered unfavorable?

\section{Statistical Analysis}

Two of the most prominent prognostication models for TBI, IMPACT ${ }^{34}$ and CRASH ${ }^{23}$ were compared to the prognostic capacities of GWR and densitometric parameters to evaluate the efficacy of the proposed parameters. Furthermore, the densitometric parameters were incorporated into the IMPACT and CRASH models to further improve the predictive performance of the models.

Due to the relatively small size of the cohorts, nonparametric analyses were performed, and the data are presented as the median (interquartile range [IQR]). The Mann-Whitney U-test and chi-square test were performed to analyze the continuous and categorical variables, respectively, and identify significant differences in the CT 
parameters (GWR, dGx, and dWx) between the two outcome groups. A difference was considered significant at a 2 -sided $p$ value $<0.05$. The prognostic capacities of the statistically significant parameters were further evaluated by analyzing a receiver operating characteristic (ROC) curve. The optimal thresholds for the parameters used to predict patient outcomes were defined as the point on the ROC curve at which the maximal Youden's index was obtained. All statistical analyses were performed using commercial software (IBM SPSS version 24, IBM Corp.). Multivariate logistic regression analyses were performed to adjust the IMPACT and CRASH models for densitometric parameters.

\section{Results}

\section{Demographics}

Noncontrast CT images and clinical information obtained from 300 patients with TBI were used in the analyses. In total, 100 patients (33.3\%) had an unfavorable outcome, 200 patients $(66.6 \%)$ were male, and the median patient age was 55.5 years (IQR 16-73 years). A significant difference in age was observed between the outcome groups. According to the radiological findings, subdural and intraventricular hemorrhages were more frequently observed in the unfavorable group than in the favorable group. The baseline demographics and clinical characteristics of the patients are summarized in Table 1.

\section{Predictive Power of CT Parameters}

Lower proportions of both normal deep GM (dGx) and normal WM $(\mathrm{dWx})$ were strongly correlated with worse outcomes (Table 2). The worse outcome group exhibited a significant decrease in both $\mathrm{dGx}$ and $\mathrm{dWx}$, indicating the presence of a severe ischemic insult or a lesion involved in decreasing the proportions of deep GM (dGx) and WM (dWx). Significant differences in dGx were not observed between the adult and geriatric groups, whereas dWx consistently and significantly differed across all age groups (Table 3 ). In contrast, none of the GWR parameters showed a meaningful difference between the favorable and unfavorable groups.

The power of the densitometric parameters in predicting the prognostic outcome was further assessed via ROC analyses (Fig. 2). The dWx parameter exhibited the highest prognostic capacity in all subjects (Fig. 2A). The decrease in the combined GM and WM density, which is depicted as a reduced $\mathrm{dGx}+\mathrm{dWx}$, exhibited the highest predictive power for worse outcomes in the pediatric subjects (Fig. 2B). In the adult and geriatric subjects, dWx effectively distinguished subjects with unfavorable outcomes (Fig. 2C and D). In contrast to the densitometric parameters, the GWRs did not effectively distinguish the outcome groups (Fig. 3). The area under the curve (AUC) values for the densitometric parameters and GWRs are summarized in Table 3.

\section{Predictive Power of TBI Prognostic Models}

The predictive capacity of the existing TBI prognostic models (i.e., IMPACT and CRASH models) were compared to that of the densitometric parameters (Table 3).
TABLE 1. Baseline demographics and clinical characteristics of the 300 patients

\begin{tabular}{|c|c|c|c|c|}
\hline Characteristic & Total & Favorable & Unfavorable & $\begin{array}{c}p \\
\text { Value* }\end{array}$ \\
\hline No. of patients & 300 & 200 & 100 & \\
\hline Age (mos) & & & & $<0.001$ \\
\hline Median & 55.5 & 49 & 66 & \\
\hline IQR & $16-73$ & $14-70$ & $45-76.8$ & \\
\hline Age group, $\mathrm{n}(\%)$ & & & & $<0.001$ \\
\hline Pediatric & $92(30.7)$ & $76(38.0)$ & $16(16.0)$ & \\
\hline Adult & $94(31.3)$ & $61(30.5)$ & $33(33.0)$ & \\
\hline Geriatric & $114(38.0)$ & $63(31.5)$ & $51(51.0)$ & \\
\hline Sex, n (\%) & & & & 0.06 \\
\hline Male & $200(66.7)$ & $126(63.0)$ & $74(74.0)$ & \\
\hline Female & $100(33.3)$ & $74(37.0)$ & $26(26.0)$ & \\
\hline Cause of exam, n (\%) & & & & $<0.001$ \\
\hline MVA & $47(15.7)$ & $33(16.5)$ & $14(14.0)$ & \\
\hline Fall & $109(36.3)$ & $68(34.0)$ & $41(41.0)$ & \\
\hline Collision & $13(4.3)$ & $10(5.0)$ & $3(3.0)$ & \\
\hline $\begin{array}{l}\text { HA/vomiting/ } \\
\text { nausea/ } \\
\text { confusion }\end{array}$ & $55(18.3)$ & $44(22.0)$ & $11(11.0)$ & \\
\hline Seizure & $30(10.0)$ & $25(12.5)$ & $5(5.0)$ & \\
\hline Others & $46(15.3)$ & $20(10.0)$ & $26(26.0)$ & \\
\hline \multicolumn{5}{|l|}{ Imaging findings, $\mathrm{n}(\%)$} \\
\hline $\begin{array}{l}\text { Epidural hemor- } \\
\text { rhage }\end{array}$ & $37(12.3)$ & $26(13.0)$ & $11(11.0)$ & 0.62 \\
\hline $\begin{array}{l}\text { Subdural hemor- } \\
\text { rhage }\end{array}$ & $140(46.7)$ & $80(40.0)$ & $60(60.0)$ & 0.001 \\
\hline $\begin{array}{l}\text { Intracerebral hem- } \\
\text { orrhage }\end{array}$ & $55(18.3)$ & $32(16.0)$ & $23(23.0)$ & 0.14 \\
\hline $\begin{array}{r}\text { Intraventricular } \\
\text { hemorrhage }\end{array}$ & $24(8.0)$ & $11(5.5)$ & $13(13.0)$ & 0.02 \\
\hline $\begin{array}{l}\text { Subarachnoid } \\
\text { hemorrhage }\end{array}$ & $54(18.0)$ & $30(15.0)$ & $24(24.0)$ & 0.06 \\
\hline Cerebral contusion & 38 (12.7) & $23(11.5)$ & $15(15.0)$ & 0.39 \\
\hline Hypodense lesions & $69(23.0)$ & $41(20.5)$ & $28(28.0)$ & 0.15 \\
\hline Midline shift $>5 \mathrm{~mm}$ & $24(8.0)$ & $14(7.0)$ & $10(10.0)$ & 0.37 \\
\hline Skull fracture & $44(14.7)$ & $28(14.0)$ & $16(16.0)$ & 0.64 \\
\hline
\end{tabular}

$\mathrm{HA}=$ headache; $\mathrm{MVA}=$ motor vehicle accident.

* Based on the results of a chi-square test for categorical variables and a Mann-Whitney U-test for continuous variables.

IMPACT and CRASH models showed adequate and reliable performance in the pediatric and geriatric groups, but not in the adult group. The densitometric parameters, particularly $\mathrm{dWx}+\mathrm{dGx}$, outperformed the IMPACT and CRASH models, with the exception of the pediatric population (IMPACT core model). The highest overall predictive performance was achieved by the densitometry-augmented IMPACT model (i.e., dGx + dWx-incorporated IMPACT model).

Regarding the densitometric parameters showing statistically significant differences, the optimal cutoff points 
TABLE 2. Assessment of CT parameters in the favorable and unfavorable outcome groups

\begin{tabular}{|c|c|c|c|c|c|c|c|c|c|}
\hline & \multicolumn{3}{|c|}{ Densitometric Parameters, $\%$} & \multicolumn{6}{|c|}{ GWR } \\
\hline & $d W x$ & $d G x$ & $d W x+d G x$ & CN/PLIC & $\mathrm{PU} / \mathrm{CC}$ & PU/PLIC & $\mathrm{GWR}_{\text {basal ganglia }}$ & $\mathrm{GWR}_{\text {cerebrum }}$ & Avg GWR \\
\hline \multicolumn{10}{|l|}{ All patients } \\
\hline \multicolumn{10}{|c|}{ Favorable $(n=200)$} \\
\hline Median & 27.21 & 21.33 & 48.89 & 1.26 & 1.26 & 1.26 & 1.25 & 1.27 & 1.27 \\
\hline IQR & $24.14-29.21$ & $17.68-24.35$ & $43.39-52.17$ & $1.17-1.34$ & $1.20-1.32$ & $1.18-1.35$ & $1.21-1.31$ & $1.19-1.34$ & $1.23-1.31$ \\
\hline \multicolumn{10}{|c|}{ Unfavorable $(n=100)$} \\
\hline Median & 21.28 & 18.95 & 39.93 & 1.24 & 1.26 & 1.26 & 1.25 & 1.27 & 1.26 \\
\hline IQR & $16.93-24.06$ & $13.32-23.58$ & $33.29-45.55$ & 1.17-1.32 & $1.18-1.36$ & $1.20-1.35$ & $1.20-1.33$ & $1.19-1.38$ & $1.20-1.33$ \\
\hline$p$ value & $<0.001$ & 0.004 & $<0.001$ & 0.52 & 0.93 & 0.62 & 0.75 & 0.44 & 0.76 \\
\hline \multicolumn{10}{|c|}{ Pediatric patients } \\
\hline \multicolumn{10}{|c|}{ Favorable $(n=76)$} \\
\hline Median & 28.97 & 23.85 & 51.99 & 1.26 & 1.25 & 1.30 & 1.25 & 1.24 & 1.25 \\
\hline IQR & $26.6-31.21$ & $21.44-27.89$ & $49.04-55.68$ & $1.17-1.34$ & $1.20-1.31$ & $1.22-1.37$ & $1.22-1.32$ & $1.15-1.31$ & $1.22-1.30$ \\
\hline \multicolumn{10}{|c|}{ Unfavorable $(n=16)$} \\
\hline Median & 22.82 & 17.30 & 42.16 & 1.30 & 1.31 & 1.34 & 1.33 & 1.25 & 1.28 \\
\hline IQR & $17.14-26.29$ & $12.24-24.02$ & $32.99-46.54$ & $1.21-1.42$ & $1.23-1.50$ & $1.29-1.41$ & $1.26-1.38$ & $1.19-1.31$ & $1.25-1.32$ \\
\hline$p$ value & $<0.001$ & 0.002 & $<0.001$ & 0.33 & 0.13 & 0.12 & 0.06 & 0.6 & 0.11 \\
\hline \multicolumn{10}{|l|}{ Adult patients } \\
\hline \multicolumn{10}{|c|}{ Favorable $(n=61)$} \\
\hline Median & 27.52 & 20.32 & 48.31 & 1.26 & 1.25 & 1.25 & 1.25 & 1.26 & 1.25 \\
\hline IQR & $24.31-28.81$ & $18.35-22.66$ & $44.55-50.5$ & $1.18-1.35$ & $1.20-1.33$ & $1.18-1.34$ & $1.19-1.31$ & $1.18-1.31$ & $1.21-1.30$ \\
\hline \multicolumn{10}{|c|}{ Unfavorable $(n=33)$} \\
\hline Median & 22.11 & 18.84 & 41.38 & 1.25 & 1.28 & 1.27 & 1.25 & 1.26 & 1.26 \\
\hline IQR & $17.63-25.26$ & $14.6-25.11$ & $34.36-49.27$ & $1.14-1.32$ & $1.21-1.36$ & $1.20-1.35$ & $1.22-1.31$ & $1.19-1.37$ & $1.21-1.32$ \\
\hline$p$ value & $<0.001$ & 0.49 & 0.003 & 0.57 & 0.29 & 0.44 & 0.96 & 0.39 & 0.75 \\
\hline \multicolumn{10}{|c|}{ Geriatric patients } \\
\hline \multicolumn{10}{|c|}{ Favorable $(n=63)$} \\
\hline Median & 25.42 & 17.89 & 43.41 & 1.24 & 1.28 & 1.22 & 1.25 & 1.30 & 1.29 \\
\hline IQR & $22.98-27.36$ & $14.07-22.42$ & $38.78-48.2$ & $1.15-1.33$ & $1.21-1.35$ & $1.16-1.30$ & $1.21-1.31$ & $1.24-1.40$ & $1.25-1.34$ \\
\hline \multicolumn{10}{|c|}{ Unfavorable $(n=51)$} \\
\hline Median & 20.07 & 19.06 & 38.99 & 1.22 & 1.22 & 1.25 & 1.24 & 1.29 & 1.26 \\
\hline IQR & $15.77-23.2$ & $12.89-23.21$ & $33.23-41.89$ & $1.17-1.30$ & $1.16-1.36$ & $1.17-1.31$ & $1.19-1.31$ & $1.18-1.40$ & $1.19-1.36$ \\
\hline$p$ value & $<0.001$ & 0.72 & $<0.001$ & 0.6 & 0.22 & 0.3 & 0.24 & 0.33 & 0.19 \\
\hline
\end{tabular}

Avg = average.

The $p$ values were based on the results of the Mann-Whitney U-test.

on the ROC curves were derived, and the performances of the cutoff points in predicting the outcomes were further evaluated (Table 4). In all patients, the dWx showed the highest net predictive value. Specifically, the density normality of the WM was particularly indicative of the outcomes in the adult and geriatric patients, whereas for the pediatric patients, the density normality of both the GM and WM was associated with the outcome.

\section{Observations}

The CT images and density distribution of the typical cases in which dWx and dGx showed typical values are shown in Fig. 4. Patients with unfavorable outcomes exhibited decreased $\mathrm{dGx}$ and $\mathrm{dWx}$, resulting in a leftward shift of the density distributions.

\section{Discussion}

Secondary insults following TBI influence the treatment strategy; thus, a timely assessment of the injury severity is essential, and methods designed to enhance the diagnostic and prognostic capacities of noncontrast CT for secondary ischemic-edematous insults after TBI are urgently needed. The GWR is used to quantify the extent of these insults following cardiac arrest. ${ }^{37}$ Given the reported efficacy of GWR, 5,21,27,33 this study applied the GWR to patients with TBI in different age groups and found that $1)$ the GWR is not associated with outcomes ( $p>0.05)$, and 2) the density normality of both the deep GM and the whole WM (particularly the latter) predicts the outcome. Compared to existing TBI prognostication models and the GWR, the densitometric parameters exhibited the 
TABLE 3. AUCs of the densitometric parameters and GWRs in predicting an unfavorable outcome

\begin{tabular}{|c|c|c|c|c|}
\hline & \multicolumn{4}{|c|}{$\mathrm{AUC}(95 \% \mathrm{Cl})$} \\
\hline & All Patients $(n=300)$ & Pediatric Patients $(n=92)$ & Adult Patients $(n=94)$ & Geriatric Patients $(n=114)$ \\
\hline \multicolumn{5}{|l|}{ Densitometric parameters } \\
\hline$d W x$ & $0.844(0.796-0.892)$ & $0.814(0.699-0.929)$ & $0.792(0.691-0.894)$ & $0.885(0.828-0.943)$ \\
\hline $\mathrm{dGx}$ & $0.601(0.530-0.672)$ & $0.751(0.602-0.900)$ & $0.543(0.403-0.684)$ & $0.510(0.400-0.621)$ \\
\hline$d W x+d G x$ & $0.783(0.727-0.839)$ & $0.881(0.778-0.984)$ & $0.684(0.560-0.807)$ & $0.720(0.626-0.814)$ \\
\hline \multicolumn{5}{|l|}{ GWRs } \\
\hline CN/PLIC & $0.523(0.453-0.593)$ & $0.585(0.417-0.752)$ & $0.536(0.411-0.662)$ & $0.529(0.421-0.636)$ \\
\hline $\mathrm{PU} / \mathrm{CC}$ & $0.503(0.428-0.578)$ & $0.631(0.438-0.824)$ & $0.567(0.441-0.693)$ & $0.568(0.459-0.677)$ \\
\hline PU/PLIC & $0.518(0.447-0.588)$ & $0.635(0.495-0.774)$ & $0.549(0.424-0.673)$ & $0.557(0.449-0.665)$ \\
\hline $\mathrm{GWR}_{\text {basal ganglia }}$ & $0.511(0.438-0.585)$ & $0.665(0.491-0.839)$ & $0.504(0.379-0.628)$ & $0.564(0.455-0.674)$ \\
\hline $\mathrm{GWR}_{\text {cerebrum }}$ & $0.528(0.455-0.600)$ & $0.546(0.401-0.690)$ & $0.554(0.428-0.680)$ & $0.553(0.443-0.664)$ \\
\hline Avg GWR & $0.511(0.436-0.586)$ & $0.641(0.484-0.798)$ & $0.520(0.392-0.648)$ & $0.572(0.458-0.685)$ \\
\hline \multicolumn{5}{|l|}{ TBI prognostic model } \\
\hline IMPACT core model & $0.673(0.603-0.742)^{*}$ & $0.946(0.839-1)^{*}$ & $0.538(0.416-0.660)$ & $0.632(0.527-0.736)$ \\
\hline IMPACT extended model & $0.714(0.648-0.781)^{\star}$ & $0.839(0.570-1)^{*}$ & $0.636(0.520-0.751)$ & $0.673(0.570-0.776)$ \\
\hline IMPACT densitometric model & $0.881(0.836-0.926)^{*}$ & $0.833(0.694-0.972)^{*}$ & $0.837(0.746-0.927)$ & $0.919(0.872-0.967)$ \\
\hline CRASH basic model & $0.736(0.677-0.796)$ & $0.766(0.616-0.917)$ & $0.587(0.467-0.707)$ & $0.735(0.642-0.828)$ \\
\hline CRASH CT model & $0.733(0.672-0.794)$ & $0.781(0.652-0.910)$ & $0.570(0.447-0.693)$ & $0.759(0.669-0.849)$ \\
\hline CRASH densitometric model & $0.865(0.820-0.911)$ & $0.873(0.778-0.969)$ & $0.795(0.692-0.898)$ & $0.898(0.841-0.954)$ \\
\hline
\end{tabular}

The $p$ values were based on the results of the Mann-Whitney U-test. Boldface type denotes AUCs with a significance level of $p<0.05$.

${ }^{*}$ Patients $<14$ years old were excluded (favorable $=28$, unfavorable $=3$ ).
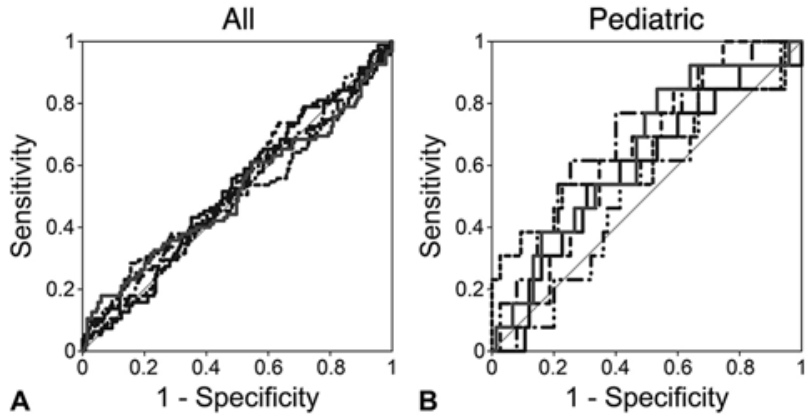

Adult
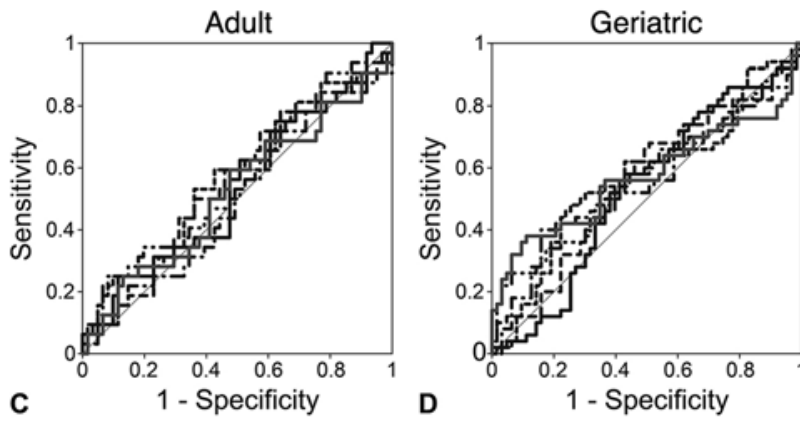

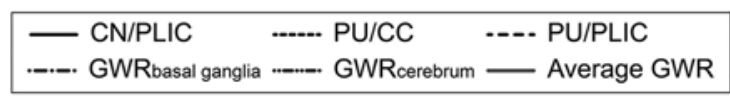

FIG. 3. Predictive power of the GWR parameters in estimating an unfavorable outcome. ROC curves show both the sensitivity and specificity of the densitometric parameters in assessing the prognosis of unfavorable outcomes in all (A), pediatric (B), adult (C), and geriatric (D) patients. highest predictive capacity; furthermore, the performance of the two TBI prognostication models, i.e., IMPACT and CRASH, was significantly enhanced when the densitometric parameters were incorporated. The findings of this study warrant a detailed discussion.

\section{Prognostic Capacity of the Loss of GM and WM Differentiation on CT Images From Patients With TBI}

The efficacy of the GWR in predicting outcomes strongly depends on the decrease in the GM density, ${ }^{3,21,37}$ which is particularly likely in patients who experience cardiac arrest. More specifically, the secondary ischemic insult leads to the development of cytotoxic (or ionic) edema, which predominantly affects the GM..$^{22}$ Even if the magnitude of the cytotoxic edema is relatively weak, the GM, particularly the deep GM, is generally more susceptible to ischemic insults than the WM. ${ }^{36}$ Thus, the GWR might still be effective in predicting the outcomes of various types of brain injuries. ${ }^{5,8,9,21,27,33}$

The decreased distinction between the GM and WM on CT images after a secondary ischemic insult is the consequence of a decrease in the GM density due to cerebral edema and an increase in the WM density after ischemicedematous insults. ${ }^{21,37}$ Torbey et al. speculated that the change in the WM density arose from the distension of deep medullary veins; however, these authors also noted that the change was not homogeneous. ${ }^{37}$ Additionally, subsequent studies using similar designs did not observe significant differences in the WM density among the subject groups. ${ }^{3,21}$ The GWR could certainly lose its prognostic capacity if the WM density decreases, which is a likely scenario in patients with TBI. This study investigated the 


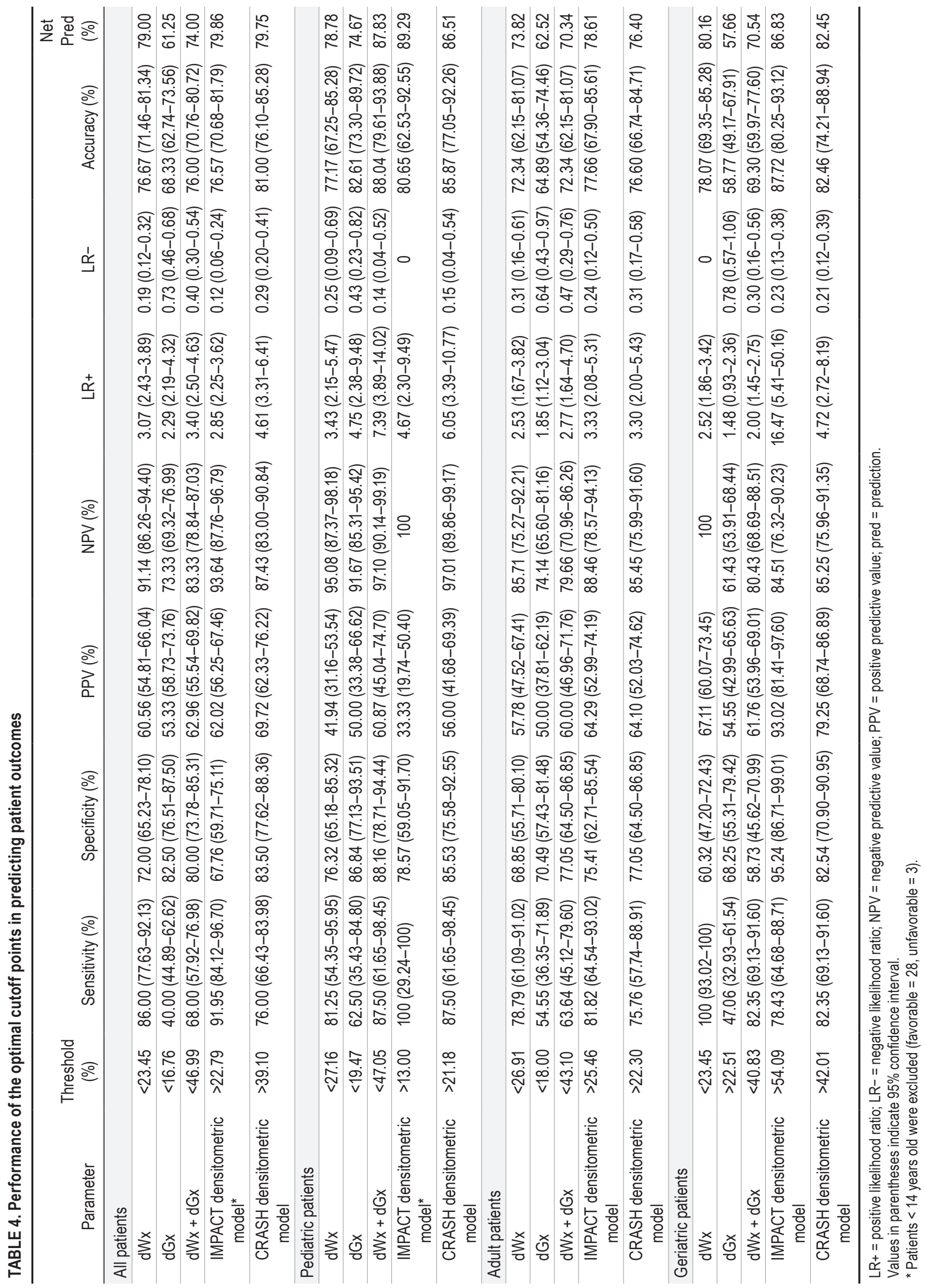


predictive value of the GWR in patients with TBI and found that the GWRs in patients with favorable and unfavorable outcomes were 1.27 (IQR 1.23-1.31) and 1.26 (IQR 1.20-1.33), respectively (Table 2), indicating that a meaningful difference in the GWR was not observed between the two patient groups. Furthermore, the decrease in the densities of both the deep GM and WM was evident in the groups with worse outcomes, which explains the ineffectiveness of the GWR.

\section{Development of Secondary Ischemic-Edematous Insults in the GM and WM After TBI and Its Association With Outcomes}

The predominant form of cerebral edema, i.e., cytotoxic or vasogenic insult, during the early phase of TBI remains controversial. ${ }^{20}$ The development of ischemic-edematous insults during early TBI has been recently shown to be more complicated than previously believed.${ }^{19} \mathrm{Lu}$ and Lei investigated the time course of cerebral edema after inducing TBI in rats and found that vasogenic edema first appeared at the injury site in the brain, followed by cytotoxic edema. At the uninjured sites, cytotoxic edema was followed by vasogenic edema. ${ }^{19}$ Although the predominant form of edema in patients with TBI is cytotoxic edema, ${ }^{4,20,44}$ cytotoxic and vasogenic edema undoubtedly coexist, and vasogenic edema may play an important role in the worsening of secondary injury during the early phase of TBI. ${ }^{25,31}$

Cytotoxic edema is commonly associated with ischemia and mainly affects the GM. In contrast, vasogenic edema mainly affects the WM, and although it often manifests as regional low attenuation, vasogenic edema easily spreads to other parts of the brain via the WM. ${ }^{13,22}$ This particular vulnerability of the WM to ischemic-edematous insults was also reported in patients with intracerebral hemorrhage, ${ }^{12}$ which shares common effects and mechanisms with TBI. ${ }^{24}$ Therefore, during the early phase of TBI, when cytotoxic and vasogenic edema coexist, the density of both the GM and WM may decrease. Thus, the density normality indices of the GM and WM (depicted as dGx and dWx, respectively), as defined by the proportions of normal GM and WM on CT images, might be significantly affected by simultaneous GM-WM changes. Following extensive cytotoxic edema, the dGx decreases, and a vasogenic insult results in a decrease in dWx (Table 2).

\section{Practical Implications of Decreased Density Normality in the GM and WM}

Previous attempts to assess the outcomes after ischemic-edematous insult using a CT analysis involved the identification of edema. ${ }^{41}$ While this approach is intuitive, the changes in the tissue density following an ischemicedematous insult may be too subtle to detect on CT images, and the density of the brain tissue is often heterogeneous, even in a single structure. ${ }^{3}$ These characteristics may hinder the accurate assessment of the extent of cerebral edema, which decreases the prognostic capacity of the derived parameters for the outcome. The quantitative, densitometric approach forgoes the accurate identification of cerebral edema, which is highly vulnerable to the subjective interpretation of the assessor. Instead, this approach derives the density normality of the deep GM and WM, i.e., the proportion of the brain tissue that is less susceptible to ischemic-edematous insults. The use of the deep GM and WM density normality indices revealed that lower proportions of normal deep GM and WM (approximately $17 \%$ and $23 \%$, respectively) indicate a worse outcome. When the combined proportion of normal deep GM and WM was less than $47 \%$, the net prediction of a worse outcome reached $74 \%$ (Table 4). Therefore, both the deep GM and WM are susceptible to ischemic-edematous insults during the early phase of TBI, and the density normality of the deep GM and WM are potentially more useful than the ratio of the GM to WM density for predicting patient outcomes during the early phase of TBI. Additionally, and perhaps more importantly, the association between the outcomes and the density normality of the GM decreases with aging; the prognostic capacity of dGx was nearly $75 \%$ in pediatric patients, whereas this capacity was $62.5 \%$ in the adult patients and $57.7 \%$ in the geriatric patients (Fig. 3 , Table 4).

The geriatric population not only exhibits age-related WM changes but also GM changes due to small infarctions, etc. ${ }^{6,30,32}$ In general, the density of the GM and brain metabolism decrease with aging, ${ }^{30,32}$ likely explaining the lack of a significant correlation between the outcome and $\mathrm{dGx}$, which is effectively a surrogate measure of ischemicedematous insults after GM in older patients. In contrast, pediatric patients are more susceptible to the insult than adult and/or geriatric patients, ${ }^{9,11}$ which would explain the higher prognostic capacity of the combined GM-WM density normality in these populations (Table 4). Although the significance of the decrease in the normal density GM has not been investigated, this parameter could be even more important in patients younger than 2 years of age due to incomplete myelination..$^{15}$ The decreased proportion of the normal density of both the WM and GM after TBI should be further investigated in this population.

In practice, the relatively low performance of densitometric parameters for assessing the outcomes of adult patients could be compensated for by incorporating the existing prognostication models for TBI; during the acute phase of the injury, the noncontrast CT images and basic admission characteristics (e.g., age, sex, motor score, and pupil response) are often available. Although the densitometric analysis remains to be validated, this assessment of initially acquired CT images shows potential to be employed in routine practice during the early stages of TBI.

\section{Study Limitations and Suggestions}

The methods used in this study provide a quantitative assessment of TBI and have the potential to become standard methods for CT-based classification of TBI. Nevertheless, some limitations of this study should be noted. First, only short-term outcomes (acquired at the time of discharge) were evaluated, which is a less than optimal measure of the outcome after TBI. The use of different CT machines, image acquisition parameters, and manufacturers of CT equipment affect the HU values of the acquired CT images. While the methods used in this study could be applied to noncontrast CT images acquired using relatively standard scanner settings, the possible HU vari- 

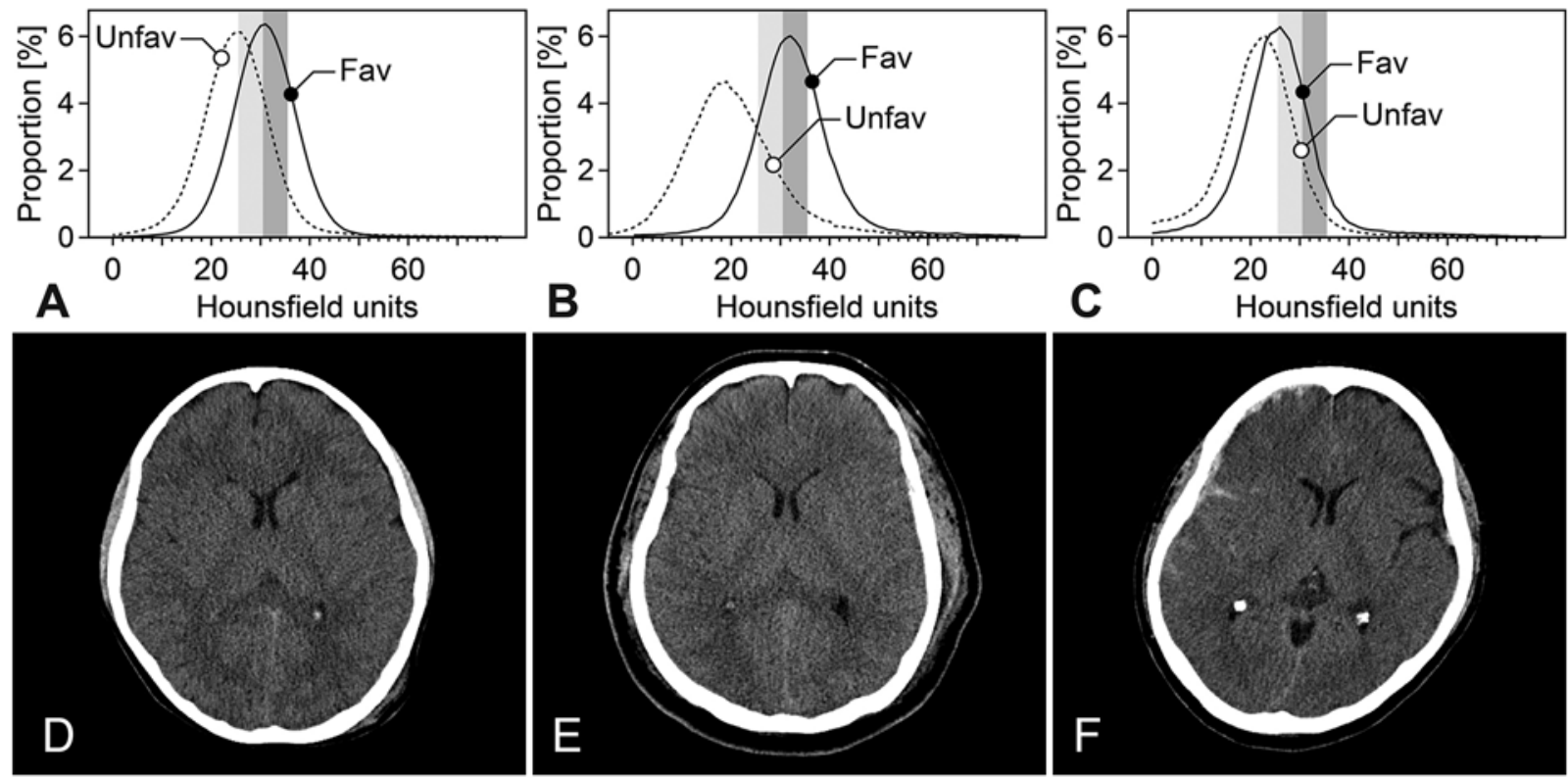

Hounsfield units
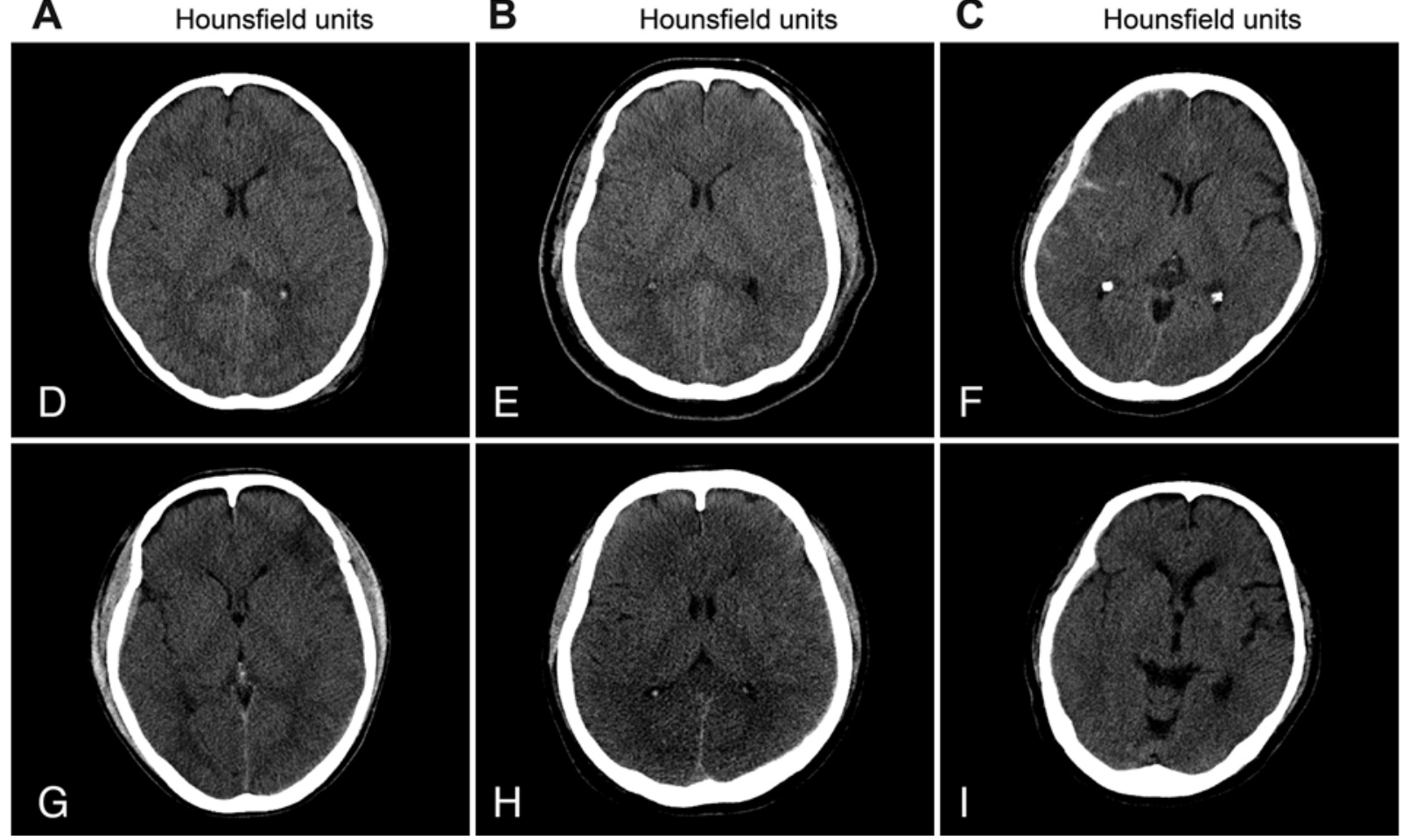

FIG. 4. Upper row: Typical density histograms for each group of patients with TBI: pediatric (A), adult (B), and geriatric (C) patients. Graphs show the distribution of the densities in patients with favorable (Fav) outcomes (black line) and unfavorable (Unfav) outcomes (dashed line). The darker gray area denotes the proportion of $\mathrm{dGx}$, whereas the lighter gray area depicts the proportion of dWx. Middle and lower rows: Typical axial CT images obtained from pediatric (D), adult (E), and geriatric (F) patients with favorable outcomes, and raw axial CT images obtained from typical patients with unfavorable outcomes in all age groups (pediatric [G], adult [H], and geriatric [l]). D: Brain CT image obtained in a pediatric patient (11-year-old boy). The scan revealed a traumatic subarachnoid hemorrhage along the sylvian fissure without an open intracranial wound. The values for dWx and dGx were 28.12 and 29.09 , respectively, which were greater than the optimal cutoff values. The patient was discharged from the hospital because his symptoms improved. E: Brain CT scan obtained in an adult patient (52-year-old man). Diffuse brain swelling was observed. Both $\mathrm{dWx}$ and $\mathrm{dGx}$ were greater than the threshold value, and the patient was discharged from the hospital with a favorable outcome. F: Brain CT scan obtained in a geriatric patient (67-year-old man). The CT revealed an acute subarachnoid hemorrhage along the right cerebral hemisphere and intracranial hemorrhage in the right frontal hemisphere. The densitometric parameters (dWx 28.20, dGx 13.81) predicted a favorable outcome. G: CT image obtained in a pediatric patient (16-year-old boy) with a significant intracranial hemorrhage. The CT scan results revealed a hemorrhage in the left frontal lobe and epidural hemorrhage in the right frontal convexity. The $\mathrm{dWx}$ was 27.15 and the $\mathrm{dGx}$ was 13.69 , both of which were lower than the optimal cutoff values. The patient showed an unfavorable outcome. H: CT image obtained in an adult patient (41-year-old man) with a contusional hemorrhage. The CT indicated a contusional hemorrhage in the left frontal and temporal lobes. The dWx was 19.39 and the dGx was 12.11, which were far lower than the optimal cutoff values. The densitometric parameter $\mathrm{dWx}$ predicted an unfavorable outcome, and the patient died. I: CT image obtained in a geriatric patient (66-year-old woman). The CT scan revealed a subarachnoid hemorrhage in the right frontotemporal area with mild compression of the lateral ventricle. The $\mathrm{dWx}$ was 19.24 , which was less than the threshold value, and the $d G x$ was 6.7. The patient had an unfavorable outcome.

ance caused by different $\mathrm{CT}$ acquisition settings should be noted. In this context, an evaluation of possible changes in the GM-WM density normality after TBI in neonates and infants may not be readily applicable, and the methods used in this study should be adequately modified. The temporal aspects of the injuries, which significantly affect the extent of the secondary injury, were not properly investigated. Further validation of the results derived using this method is needed before this method is used in clinical practice, preferably via a multicenter prospective study. Finally, the overall brain density is affected by the age of the patients. While the findings of this study implicate the overall changes in GM and WM densities as more effective predictors of the outcomes of patients with TBI 
than the GWR, a conventional quantitative measure differentiating the GM and WM, the GWR might be further enhanced by adjusting for age, thereby increasing the efficacy of the parameter for predicting functional outcomes after TBI.

\section{Conclusions}

Both GM and WM are susceptible to secondary ischemic injury during early TBI. A quantitative, densitometric analysis of noncontrast $\mathrm{CT}$ images obtained from patients with TBI was conducted to assess the density normality of GM and WM. The density normality indices dGx and dWx exhibited significant prognostic capacity for patients with TBI. The extent of the secondary injuries during early TBI was evaluated by deriving the proportions of GM and WM that were unaffected by ischemic-edematous insults. Based on the findings obtained using this approach, while adult and geriatric patients are more vulnerable to WM damage, pediatric patients are particularly vulnerable to GM injury. The calculation of the density normality is rapid and fully automated. The methods and findings of this study deserve attention and warrant further validation.

\section{Acknowledgments}

This research was supported by a grant from the Korea Health Technology R\&D Project through the Korea Health Industry Development Institute, funded by the Ministry of Health \& Welfare, Republic of Korea (grant no. HI17C1790) and by the Ministry of Science and ICT, Korea, under the Information Technology Research Center support program (IITP-2017-2016-0-00464) supervised by the Institute for Information \& Communications Technology Promotion and supported by a Korea University grant.

\section{References}

1. Brunker C, Harris R: How accurate is the AVPU scale in detecting neurological impairment when used by general ward nurses? An evaluation study using simulation and a questionnaire. Intensive Crit Care Nurs 31:69-75, 2015

2. Cala LA, Thickbroom GW, Black JL, Collins DW, Mastaglia FL: Brain density and cerebrospinal fluid space size: CT of normal volunteers. AJNR Am J Neuroradiol 2:41-47, 1981

3. Choi SP, Park HK, Park KN, Kim YM, Ahn KJ, Choi KH, et al: The density ratio of grey to white matter on computed tomography as an early predictor of vegetative state or death after cardiac arrest. Emerg Med J 25:666-669, 2008

4. Donkin JJ, Vink R: Mechanisms of cerebral edema in traumatic brain injury: therapeutic developments. Curr Opin Neurol 23:293-299, 2010

5. Gentsch A, Storm C, Leithner C, Schroeder T, Ploner CJ, Hamm B, et al: Outcome prediction in patients after cardiac arrest: a simplified method for determination of gray-white matter ratio in cranial computed tomography. Clin Neuroradiol 25:49-54, 2015

6. Gold G, Kövari E, Herrmann FR, Canuto A, Hof PR, Michel JP, et al: Cognitive consequences of thalamic, basal ganglia, and deep white matter lacunes in brain aging and dementia. Stroke 36:1184-1188, 2005

7. Gruen P: Handbook of head trauma: acute care to recovery. Neurosurgery 34:938-939, 1994

8. Heinz UE, Rollnik JD: Outcome and prognosis of hypoxic brain damage patients undergoing neurological early rehabilitation. BMC Res Notes 8:243, 2015

9. Huang BY, Castillo M: Hypoxic-ischemic brain injury: imaging findings from birth to adulthood. Radiographics 28:417-439, 2008

10. Hyder AA, Wunderlich CA, Puvanachandra P, Gururaj G, Kobusingye OC: The impact of traumatic brain injuries: a global perspective. NeuroRehabilitation 22:341-353, 2007

11. Kim H, Kim GD, Yoon BC, Kim K, Kim BJ, Choi YH, et al: Quantitative analysis of computed tomography images and early detection of cerebral edema for pediatric traumatic brain injury patients: retrospective study. BMC Med 12:186, 2014

12. Kim H, Yang X, Choi YH, Yoon BC, Kim K, Kim DJ: Abilities of a densitometric analysis of computed tomography images and hemorrhagic parameters to predict outcome favorability in patients with intracerebral hemorrhage. Neurosurgery 88:226-236, 2018

13. Kim JJ, Gean AD: Imaging for the diagnosis and management of traumatic brain injury. Neurotherapeutics 8:39-53, 2011

14. Kim SH, Choi SP, Park KN, Youn CS, Oh SH, Choi SM: Early brain computed tomography findings are associated with outcome in patients treated with therapeutic hypothermia after out-of-hospital cardiac arrest. Scand J Trauma Resusc Emerg Med 21:57, 2013

15. Knickmeyer RC, Gouttard S, Kang C, Evans D, Wilber K, Smith JK, et al: A structural MRI study of human brain development from birth to 2 years. J Neurosci 28:12176-12182, 2008

16. Lee B, Newberg A: Neuroimaging in traumatic brain imaging. NeuroRx 2:372-383, 2005

17. Lee BK, Jeung KW, Lee HY, Jung YH, Lee DH: Combining brain computed tomography and serum neuron specific enolase improves the prognostic performance compared to either alone in comatose cardiac arrest survivors treated with therapeutic hypothermia. Resuscitation 84:1387-1392, 2013

18. Lee BK, Jeung KW, Song KH, Jung YH, Choi WJ, Kim SH, et al: Prognostic values of gray matter to white matter ratios on early brain computed tomography in adult comatose patients after out-of-hospital cardiac arrest of cardiac etiology. Resuscitation 96:46-52, 2015

19. Lu H, Lei X: The apparent diffusion coefficient does not reflect cytotoxic edema on the uninjured side after traumatic brain injury. Neural Regen Res 9:973-977, 2014

20. Lucking SE, Maffei FA, Tamburro RF, Thomas NJ: Pediatric Critical Care Study Guide: Text and Review. London: Springer London, 2012

21. Metter RB, Rittenberger JC, Guyette FX, Callaway CW: Association between a quantitative CT scan measure of brain edema and outcome after cardiac arrest. Resuscitation 82:1180-1185, 2011

22. Naidich TP, Castillo M, Cha S, Smirniotopoulos JG (eds): Imaging of the Brain: Expert Radiology Series, ed 1. Philadelphia: Saunders Elsevier-Health Sciences Division, 2012

23. Perel P, Arango M, Clayton T, Edwards P, Komolafe E, Poccock S, et al: Predicting outcome after traumatic brain injury: practical prognostic models based on large cohort of international patients. BMJ 336:425-429, 2008

24. Powers WJ: Intracerebral hemorrhage and head trauma: common effects and common mechanisms of injury. Stroke 41 (10 Suppl):S107-S110, 2010

25. Reithmeier T, Speder B, Pakos P, Brinker G, Löhr M, Klug $\mathrm{N}$, et al: Delayed bilateral craniectomy for treatment of traumatic brain swelling in children: case report and review of the literature. Childs Nerv Syst 21:249-254, 2005

26. Rusnak M: Traumatic brain injury: giving voice to a silent epidemic. Nat Rev Neurol 9:186-187, 2013

27. Scheel M, Storm C, Gentsch A, Nee J, Luckenbach F, Ploner $\mathrm{CJ}$, et al: The prognostic value of gray-white-matter ratio in cardiac arrest patients treated with hypothermia. Scand $\mathbf{J}$ Trauma Resusc Emerg Med 21:23, 2013

28. Schneider W, Bortfeld T, Schlegel W: Correlation between 
CT numbers and tissue parameters needed for Monte Carlo simulations of clinical dose distributions. Phys Med Biol 45:459-478, 2000

29. Shekhar C, Gupta LN, Premsagar IC, Sinha M, Kishore J: An epidemiological study of traumatic brain injury cases in a trauma centre of New Delhi (India). J Emerg Trauma Shock 8:131-139, 2015

30. Shen X, Liu H, Hu Z, Hu H, Shi P: The relationship between cerebral glucose metabolism and age: report of a large brain PET data set. PLoS One 7:e51517, 2012

31. Simard JM, Kent TA, Chen M, Tarasov KV, Gerzanich V: Brain oedema in focal ischaemia: molecular pathophysiology and theoretical implications. Lancet Neurol 6:258-268, 2007

32. Sowell ER, Peterson BS, Thompson PM, Welcome SE, Henkenius AL, Toga AW: Mapping cortical change across the human life span. Nat Neurosci 6:309-315, 2003

33. Starling RM, Shekdar K, Licht D, Nadkarni VM, Berg RA, Topjian AA: Early head CT findings are associated with outcomes after pediatric out-of-hospital cardiac arrest. Pediatr Crit Care Med 16:542-548, 2015

34. Steyerberg EW, Mushkudiani N, Perel P, Butcher I, Lu J, McHugh GS, et al: Predicting outcome after traumatic brain injury: development and international validation of prognostic scores based on admission characteristics. PLoS Med 5:e165, 2008

35. Stoel BC, Marquering HA, Staring M, Beenen LF, Slump $\mathrm{CH}$, Roos YB, et al: Automated brain computed tomographic densitometry of early ischemic changes in acute stroke. J Med Imaging (Bellingham) 2:014004, 2015

36. Todd NV, Picozzi P, Crockard A, Russell RW: Duration of ischemia influences the development and resolution of ischemic brain edema. Stroke 17:466-471, 1986

37. Torbey MT, Selim M, Knorr J, Bigelow C, Recht L: Quantitative analysis of the loss of distinction between gray and white matter in comatose patients after cardiac arrest. Stroke 31:2163-2167, 2000

38. Voller B, Auff E, Schnider P, Aichner F: To do or not to do? Magnetic resonance imaging in mild traumatic brain injury. Brain Inj 15:107-115, 2001

39. Wendt R: The mathematics of medical imaging: a beginner's guide. J Nucl Med 51:1987, 2010

40. Wu O, Batista LM, Lima FO, Vangel MG, Furie KL, Greer
DM: Predicting clinical outcome in comatose cardiac arrest patients using early noncontrast computed tomography. Stroke 42:985-992, 2011

41. Yamamura H, Morioka T, Yamamoto T, Mizobata Y: Head computed tomographic measurement as a predictor of outcome in patients with subdural hematoma with cerebral edema. Scand J Trauma Resusc Emerg Med 24:83, 2016

42. Zasler ND, Katz DI, Ross D, Zafonte D: Brain Injury Medicine, ed 2: Principles and Practice. Berlin: Springer, 2012

43. Zech WD, Jackowski C, Buetikofer Y, Kara L: Characterization and differentiation of body fluids, putrefaction fluid, and blood using Hounsfield unit in postmortem CT. Int J Legal Med 128:795-802, 2014

44. Zhang C, Chen J, Lu H: Expression of aquaporin-4 and pathological characteristics of brain injury in a rat model of traumatic brain injury. Mol Med Rep 12:7351-7357, 2015

\section{Disclosures}

The authors report no conflict of interest concerning the materials or methods used in this study or the findings specified in this paper.

\section{Author Contributions}

Conception and design: DJ Kim, H Kim. Acquisition of data: K Kim. Analysis and interpretation of data: DJ Kim, H Kim, YT Kim. Drafting the article: H Kim. Critically revising the article: all authors. Reviewed submitted version of manuscript: all authors. Approved the final version of the manuscript on behalf of all authors: DJ Kim. Statistical analysis: YT Kim. Study supervision: DJ Kim.

\section{Supplemental Information}

Current Affiliations

Dr. Yoon: Department of Radiology, Massachusetts General Hospital, Boston, Massachusetts.

\section{Correspondence}

Dong-Joo Kim: Korea University, Seoul, South Korea. dongjookim@korea.ac.kr. 